\title{
Desempenho agronômico de cultivares de alho vernalizado e não vernalizado na região Sudeste de Mato Grosso
}

\author{
Evandro Marcos Biesdorf ${ }^{1}$, Jefferson Santana Silva ${ }^{2}$, Elivelton Maciel Biesdorf ${ }^{2}$, Osvaldo José \\ de Oliveira ${ }^{2}$, Murilo Viotto Del Conte ${ }^{1}$
}

\footnotetext{
${ }^{1}$ Universidade Federal de Viçosa-MG, Brasil, E-mail: evandromarcospva@ hotmail.com, muriloviottodelconte@ hotmail.com

${ }^{2}$ Instituto Federal do Mato Grosso, Campus São Vicente, Santo Antônio do Leverger-MT, Brasil, E-mail: jeffersonsantana@agronomo.eng.br, elivelton.biesdorf@agronomo.eng.br, ojolivei@yahoo.com.br
}

Recebido: 21/08/2015; Aceito: 08/10/2015.

\section{RESUMO}

Nos últimos anos, o uso da vernalização tem possibilitado o cultivo de alho nobre (Allium sativum L.) em regiões nas quais as condições de fotoperíodo e temperatura não satisfazem as exigências da cultura. Neste contexto, este estudo teve como objetivo avaliar o desempenho agronômico de 13 cultivares de alho vernalizados e não vernalizados na região sudeste de Mato Grosso. O experimento foi realizado em Santo Antônio do Leverger, MT, de maio a outubro de 2012. Os cultivares de alho não vernalizados testadas foram: Hozan L.V, Hozan, Amarante L.V, Chinês Real, Gigante do Núcleo, Amarante, Caturra, Curitibanos Gigante Roxo. Os cultivares de alho Quitéria L.V, Caçador, Quitéria, e Jonas foram submetidos a um período de vernalização de pré-plantio à 5 oC, por 54 dias e umidade relativa de $70 \%$. O delineamento experimental utilizado foi o de blocos casualizados com 13 tratamentos (cultivares) e quatro repetições. Foram avaliados: diâmetro médio de bulbos ( $\mathrm{mm}$ ), massa média de bulbos ( $\mathrm{g}$ ) e produtividade total de bulbos $\left(\mathrm{kg} \mathrm{ha}^{-1}\right)$. O cultivar Quitéria L.V apresentou maior diâmetro e massa média de bulbos quando comparado com os demais cultivares de alho testados. As maiores produtividades totais de bulbos foram obtidas com os cultivares Quitéria L.V., Quitéria, Caçador, Hozan e Jonas. Com base nos resultados obtidos neste estudo recomenda-se o plantio do cultivar Quitéria L.V. para a região sudeste de Mato Grosso, utilizando-se a vernalização pré-plantio do bulbilho-semente.

Palavras-chave: Allium sativum, vernalização, produtividade de bulbos.

\section{Performance of garlic cultivars vernalizated and non-vernalizated in Southeast of the Mato Grosso}

\begin{abstract}
In recent years, the use of vernalization has enabled the cropping of garlic (Allium sativum $\mathrm{L}$.) in regions where the conditions of photoperiod and temperature does not meet the requirements of the crop. In this context, this study aimed to evaluate the agronomic performance of 13 garlic cultivars vernalized and non-vernalized in Southeastern of the Mato Grosso. The experiment was carried out in Santo Antônio do Leverger, MT, Brazil, from May to October, 2012. The garlic cultivars non-vernalized tested were: Hozan L.V, Hozan, Amarante L.V, Chinês Real, Gigante do Núcleo, Amarante, Caturra, Curitibanos and Gigante Roxo. The cultivars Garlic Quitéria L.V, Caçador, Quiteria and Jonas were subjected to vernalization to $5{ }^{\circ} \mathrm{C}$, for 54 days and relative air humidity of $70 \%$. The experimental design was randomized blocks with 13 treatments (cultivars) and four replications. The mean diameter bulbs $(\mathrm{mm})$, mean bulb weight $(\mathrm{g})$ and bulb yield $\left(\mathrm{kg} \mathrm{ha}^{-1}\right)$ were measured. The cultivar Quitéria L.V. showed greater diameter and bulb weight when compared with the other garlic cultivars. The highest bulb yield were obtained with cultivars Quitéria L.V, Quiteria, Caçador, Hozan and Jonas. Based on the results obtained in this study recommended planting cultivate Quitéria L.V for the southeast of Mato Grosso, Brazil, using vernalization pre-planting of the bulbils.
\end{abstract}

Keywords: Glycine max L., seed sizer, chlorophyll in the seed, germination, vigor. 


\section{Introdução}

O alho (Allium sativum L.) é uma das plantas mais antigas que se tem registro, cultivadas desde a Antiguidade, para muitos é considerada como um remédio para as mais diversas doenças e para outros como um condimento de excelentes propriedades de sabor e aroma (FILGUEIRA, 2003). O alho, pertencente à família Liliaceae, é uma planta assexuada com propagação através do plantio dos bulbilhos ou dentes e assim é uma importante geradora de divisas pois possibilita a criação de grande número de empregos, sobretudo no campo, devido à grande exigência de mãode-obra desde a semeadura até a comercialização, possibilitando rentabilidades para grandes, médios e pequenos produtores (VILELA; HENZ, 2000).

Atualmente a cultura do alho ocupa o quarto lugar em importância econômica dentre as hortaliças cultivadas no Brasil com uma produção de 89.285 toneladas, sendo superada apenas pelas culturas do tomate, cebola e batata (IBGE, 2015). As maiores produções de bulbos de alho no país estão localizadas nas regiões centro-oeste (40.082 toneladas na safra 2012), sul e sudeste com 39.484 e 20.065 toneladas de bulbos de alho respectivamente na safra 2012 (CONAB, 2013). Apesar de a região centro-oeste ser a maior produtora de alho, não se encontra registros do cultivo desta hortaliça para o estado de Mato Grosso. Esta produção está concentrada apenas nos estados de Goiás (35.753 toneladas) e Distrito Federal (5.132 toneladas).

$\mathrm{O}$ alho é uma cultura de clima frio, resistente a temperaturas baixas e geadas não muito intensas. No entanto, atualmente, a cultura pode ser cultivada em diferentes condições climáticas, havendo diversos cultivares adequados a cada região. $\mathrm{O}$ cultivo de alho nobre em regiões com condições de fotoperíodo e temperatura não satisfazem as exigências da cultura, como nas regiões centro-oeste e nordeste, sendo possível seu cultivo apenas com o uso da vernalização.

A vernalização altera as exigências agroclimáticas e reduz o ciclo da cultura do alho. A exposição dos bulbilhos à temperatura entre 0 e $10{ }^{\circ} \mathrm{C}$ por um a dois meses aceleram a bulbificação, substituindo as exigências climáticas iniciais (JONES; MANN, 1963). Segundo Zink (1963), o fato de utilizar o processo de vernalização antecipa a formação do bulbo, reduz o ciclo e quebra a dormência dos bulbilhos.

O alho possui grande diversidade fenotípica, de modo que o mesmo genótipo ou clone apresenta variações morfológicas em resposta às interações com os fatores edafoclimáticos, como solo, temperatura, fotoperíodo, umidade, entre outros (JONES; MANN, 1963). Desta forma, cultivares de alho recomendados para uma determinada região não servem para indicação segura para plantio em qualquer outra região.
A avaliação da adaptação de cultivares em uma determinada região pode contribuir para aumentar a produção nacional de alho e suprir parte da demanda interna do produto. Além disso, a seleção de cultivares com desempenho satisfatório para as condições locais pode causar grandes impactos sociais e econômicos para os agricultores familiares, tornando-se uma alternativa de produção.

Diante da inexistência de informações a respeito do cultivo de alho no estado de Mato Grosso, o presente estudo teve como objetivos avaliar o desempenho agronômico de 13 cultivares de alho, submetidos ou não a vernalização, e identificar os cultivares que possuem maior adaptação as condições edafoclimáticas da região sudeste do estado de Mato Grosso.

\section{Material e Métodos}

A pesquisa foi conduzida na área experimental do Instituto Federal de Educação Ciência e Tecnologia do Estado de Mato Grosso (IFMT), Campus São Vicente, localizado no município de Santo Antônio do Leverger, MT ( $15^{\circ} 49^{\prime} \mathrm{S}$ e $55^{\circ} 25^{\prime} \mathrm{W}$ com $790 \mathrm{~m}$ de altitude). O clima da região segundo a classificação de Köppen é do tipo Aw. O solo foi classificado como Latossolo Vermelho distrófico (LVd), de textura argilosa, com relevo suave ondulado a ondulado, e originalmente coberto por savana arbórea e/ou florestas de galeria.

$\mathrm{O}$ delineamento experimental utilizado foi de blocos casualizados, com quatro repetições e treze tratamentos. Cada unidade experimental foi constituída por quatro linhas de 1,0 m de comprimento, espaçadas entre si por 0,25 m (área total de 1,0 $\mathrm{m}^{2}$ ). Para eliminar o efeito de bordadura foi considerada como área útil as duas fileiras centrais, desprezando-se $0,25 \mathrm{~m}$ das extremidades laterais do canteiro e $0,25 \mathrm{~m}$ das extremidades de cada fileira de plantio.

Os bulbilhos-semente utilizados neste estudo foram adquiridos por meio de parceria realizada junto a Embrapa Hortaliças e Universidade Federal de Goiás UFG, Campus Jataí, Goiás. Os materiais genéticos provenientes da Embrapa Hortaliças foram: Quitéria L.V, Quitéria, Caçador, Hozan L.V, Hozan, Amarante L.V, Amarante, Jonas, Chinês Real, Gigante do Núcleo e os materiais provenientes da UFG foram: Gigante Roxo e Curitibanos.

Os cultivares de alho submetidos a um período de vernalização pré-plantio foram: Quitéria L.V, Caçador, Quitéria e Jonas. Os bulbilhos-semente foram acondicionados em caixas de papelão e acomodados em uma câmara fria à $5{ }^{\circ} \mathrm{C}$, por 54 dias com umidade relativa do ar de $70 \%$.

O plantio dos cultivares de alho foi realizado no dia 03 de maio de 2012, em área com sistema de irrigação por aspersão. Inicialmente, o solo foi preparado 
utilizando-se uma gradagem pesada e uma gradagem leve e, em seguida, realizou-se a construção dos canteiros com 0,20 m de altura, largura de 1,20 m e comprimento de $15 \mathrm{~m}$. A adubação de plantio foi realizada com a aplicação de $20 \mathrm{~kg} \mathrm{ha}^{-1}$ de N, $200 \mathrm{~kg} \mathrm{ha}$ ${ }^{1}$ de $\mathrm{P}_{2} \mathrm{O}_{5}, 50 \mathrm{~kg} \mathrm{ha}^{-1} \mathrm{~K}_{2} \mathrm{O}, 2 \mathrm{~kg} \mathrm{ha}^{-1}$ de boro (B) e $2 \mathrm{~kg}$ $\mathrm{ha}^{-1}$ de zinco (Zn). Em cobertura foram aplicados $50 \mathrm{~kg}$ $\mathrm{ha}^{-1}$ de $\mathrm{N}$ e $30 \mathrm{~kg} \mathrm{ha}^{-1}$ de $\mathrm{K}_{2} \mathrm{O}$; estas doses foram divididas em duas aplicações aos 30 e 50 dias após a brotação. As irrigações por aspersão foram feitas quatro vezes por semana até 20 dias antes da colheita, utilizando-se uma lâmina de água de $10 \mathrm{~mm}$.

A colheita foi realizada aos 150 dias após o plantio, na fase de senescência das plantas, quando havia em torno de quatro folhas verdes. Após a colheita, as plantas foram curadas ao sol por cinco dias. Posteriormente, as plantas foram acondicionadas em ambiente protegido, onde foram curadas por 20 dias. Após a cura, foi realizado a toalete dos bulbos, através do corte do pseudocaule à $1,0 \mathrm{~cm}$ acima do bulbo e retirada de raízes e películas sujas.

Foi avaliada a produtividade de bulbos $\left(\mathrm{kg} \mathrm{ha}^{-1}\right)$, a massa média dos bulbos (g) e o diâmetro médio dos bulbos (mm). A massa média e o diâmetro médio dos bulbos foram obtidos por meio da média de 10 bulbos por parcela.

Os dados foram submetidos à análise de variância e as médias agrupadas pelo teste de Scott \& Knott (1974) a 5\% de significância.

\section{Resultados e Discussão}

$\mathrm{O}$ diâmetro médio dos bulbos agrupou os cultivares de alho em 3 grupos de tamanhos (Tabela 1). O cultivar Quitéria L.V (vernalizado) apresentou maior diâmetro de bulbos (45,2 mm) quando comparada com os demais cultivares de alho testados. Os cultivares Caçador (vernalizado), Hozan L.V, Hozan, Jonas (vernalizado), Quitéria (vernalizado) e Amarante L.V apresentaram diâmetro médio de bulbos intermediários (média de 36,7 $\mathrm{mm}$ ), enquanto que os cultivares Curitibanos, Amarante, Caturra, Chinês Real, Gigante do Núcleo e Gigante Roxo apresentaram os menores diâmetros de bulbos (média de 28,0 mm). Esse resultado é consequência das diferenças genéticas entre os materiais utilizados. Oliveira et al. (2010) avaliando o desempenho agronômico de 14 cultivares de alho em Diamantina, MG, constataram que os cultivares Gigante do Núcleo, Chinês Real, Amarante, Caturra, Hozan e Gigante Roxo não apresentaram diferenças no diâmetro de bulbos. Trani et al. (2008) também não verificaram diferenças no diâmetro de bulbos em estudo conduzido com os cultivares Jonas, Curitibanos, Santa Catarina Roxo, Santa Catarina branco e Assai 3702 no estado de São Paulo. Por sua vez, Oliveira et al. (2003) observaram diferenças no diâmetro de bulbos entre alguns desses cultivares em estudo conduzido no município de Lavras (MG). Segundo Mota (2006), essas diferenças podem estar relacionadas a diferentes condições de temperatura, fotoperíodo e altitude.

$\mathrm{O}$ maior diâmetro de bulbos resultou em maiores respostas em produção de bulbos (Figura 1). Trani et al. (2008) também constataram que houve correlação positiva entre diâmetro e produtividade de bulbos.

Tabela 1. Diâmetro médio de bulbos dos diferentes cultivares de alho cultivados na região sudeste do estado do Mato Grosso. IFMT - Campus São Vicente, Santo Antônio de Leverger, 2012

\begin{tabular}{lc}
\hline Cultivares & $\begin{array}{c}\text { Diâmetro médio de bulbos } \\
(\mathbf{m m})\end{array}$ \\
\hline Quitéria L.V (vernalizado) & $45,2 \mathrm{a}$ \\
Caçador (vernalizado) & $39,8 \mathrm{~b}$ \\
Hozan L.V & $38,8 \mathrm{~b}$ \\
Hozan & $36,4 \mathrm{~b}$ \\
Jonas (vernalizado) & $35,8 \mathrm{~b}$ \\
Quitéria (vernalizado) & $34,7 \mathrm{~b}$ \\
Amarante L.V & $34,6 \mathrm{~b}$ \\
Curitibanos & $31,3 \mathrm{c}$ \\
Amarante & $28,7 \mathrm{c}$ \\
Caturra & $28,0 \mathrm{c}$ \\
Chinês Real & $27,3 \mathrm{c}$ \\
Gigante do Núcleo & $27,0 \mathrm{c}$ \\
Gigante Roxo & $26,0 \mathrm{c}$ \\
\hline
\end{tabular}
CV $(\%): 9,77$

Média Geral: 33,24

Médias seguidas pela mesma letra, na coluna, pertencem ao mesmo grupo pelo teste de agrupamento de Scott e Knott (1974), a 5\% de probabilidade.

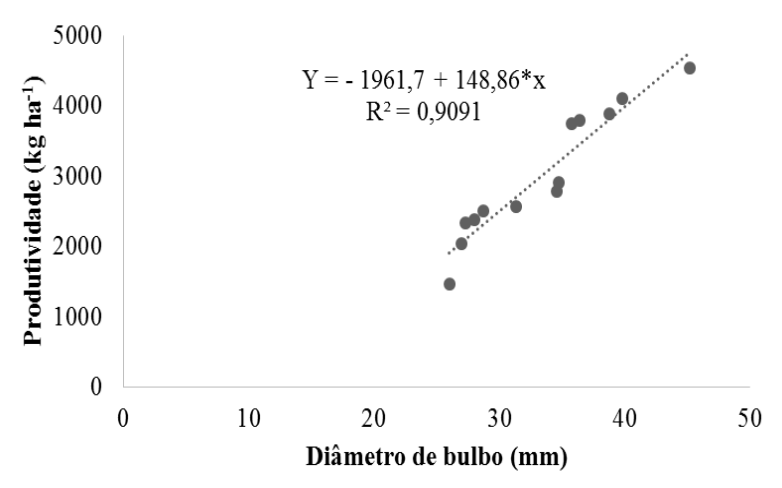

Figura 1. Correlação entre diâmetro médio de bulbos e produtividade total de bulbos para as diferentes cultivares de alho cultivadas na região sudeste do estado de Mato Grosso. IFMT - Campus São Vicente, Santo Antônio de Leverger, 2012. *: Significativo ao nível de $1 \%$ de probabilidade pelo teste t.

A massa média dos bulbos agrupou os cultivares de alho em três grupos de tamanho (Tabela 2). O cultivar Quitéria L.V (vernalizado) apresentou a maior massa de bulbo (33,5 g) quando comparado com os demais cultivares testados. Os cultivares Caçador (vernalizado), Quitéria (vernalizado), Hozan L.V., Amarante L.V, Hozan e Jonas (vernalizado) apresentaram valor 
intermediário de massa média de bulbos (média de 24,2 g), ao passo que os cultivares Chinês Real, Curitibanos, Gigante Roxo, Gigante do Núcleo, Amarante e Caturra apresentaram os menores valores de massa de bulbos (média de 15,3 g) (Tabela 2). Em estudo realizado por Oliveira et al. (2003), em Diamantina, MG, as cultivares Chinês Real e Caturra foram as que apresentaram maior massa de bulbos enquanto as cultivares Gigante Roxo, Gigante do Núcleo, Hozan e Amarante obtiveram os menores valores. A diferença na massa média de bulbos também foi reportada por Menezes et al. (1999). De acordo com esses autores, a diferença de resposta entre os cultivares é devida às condições ambientais no cultivo do alho nas diferentes regiões.

Houve correlação significativa entre a massa de bulbos e produção total de bulbos, de modo que a maior massa de bulbos resultou em maior produção de bulbos (Figura 2).

Tabela 2. Massa média de bulbos para os diferentes cultivares de alho cultivados na região sudeste do estado do Mato Grosso. IFMT - Campus São Vicente, Santo Antônio de Leverger, 2012

\begin{tabular}{lc}
\hline Cultivares & $\begin{array}{c}\text { Massa média de bulbos } \\
(\mathbf{g})\end{array}$ \\
\hline Quitéria L.V (vernalizado) & $33,5 \mathrm{a}$ \\
Caçador (vernalizado) & $27,5 \mathrm{~b}$ \\
Quitéria (vernalizado) & $26,0 \mathrm{~b}$ \\
Hozan L.V & $25,5 \mathrm{~b}$ \\
Amarante L.V & $23,0 \mathrm{~b}$ \\
Hozan & $22,5 \mathrm{~b}$ \\
Jonas (vernalizado) & $21,0 \mathrm{~b}$ \\
Chinês Real & $19,5 \mathrm{c}$ \\
Curitibanos & $18,0 \mathrm{c}$ \\
Gigante Roxo & $17,0 \mathrm{c}$ \\
Gigante do Núcleo & $14,5 \mathrm{c}$ \\
Amarante & $13,6 \mathrm{c}$ \\
Caturra & $9,5 \mathrm{c}$ \\
\hline
\end{tabular}

CV (\%): 22,43

Média Geral: 20,85

Médias seguidas pela mesma letra, na coluna, pertencem ao mesmo grupo pelo teste de agrupamento de Scott e Knott (1974), a 5\% de probabilidade.

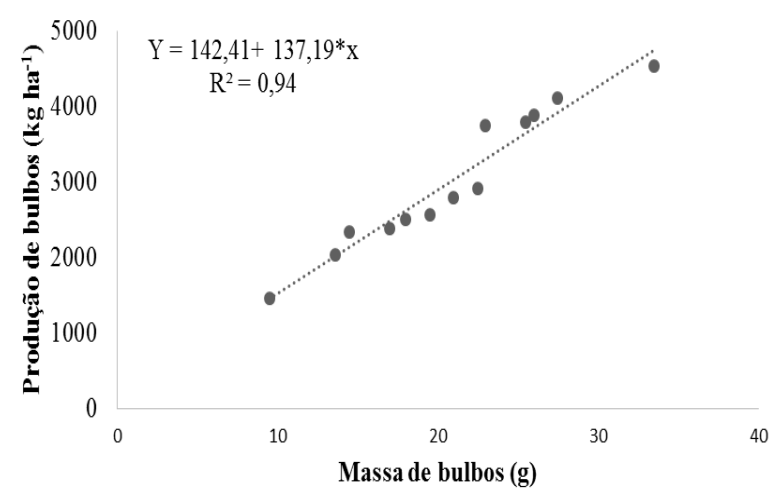

Figura 2. Correlação entre massa média de bulbos de alho e produtividade total de bulbos para os diferentes cultivares de alho cultivados na região sudeste do estado de Mato Grosso. IFMT Campus São Vicente, Santo Antônio de Leverger, 2012. *: Significativo ao nível de $1 \%$ de probabilidade pelo teste t.
A produtividade dos bulbos agrupou os cultivares de alho em três grupos de potencial de produção (Tabela 3). Os cultivares Quitéria L.V (vernalizado), Quitéria (vernalizado), Caçador (vernalizado), Hozan e Jonas (vernalizado) apresentaram as maiores produtividades de bulbos (4.011 $\mathrm{kg} \mathrm{ha}^{-1}$ ) quando comparados aos demais cultivares de alho testados. Os cultivares Curitibanos, Chinês Real, Gigante do Núcleo, Caturra, Amarante L.V e Hozan L.V. apresentaram produtividade de bulbos intermediária (produção média de $2.581 \mathrm{~kg} \mathrm{ha}^{-1}$ ), ao passo que os cultivares Amarante e Gigante Roxo apresentaram as menores produtividades de bulbos (média de $1.750 \mathrm{~kg} \mathrm{ha}^{-1}$ ) (Tabela 3). A produtividade de bulbos dos cultivares do primeiro grupo foi $229 \%$ superior à produtividade total dos cultivares pertencentes ao terceiro grupo (Amarante e Gigante Roxo). Em trabalho conduzido por Oliveira et al. (2010), em Diamantina, MG, os cultivares Chinês Real (10.650 kg ha $\left.{ }^{-1}\right)$ e Caturra $\left(9.680 \mathrm{~kg} \mathrm{ha}^{-1}\right)$ foram os que apresentaram as maiores produtividades seguidos dos cultivares Gigante do Núcleo (7.800 kg ha-1), Gigante Roxo (8.020 kg ha-1), Hozan (6.300 kg ha-1) e Amarante $\left(8.000 \mathrm{~kg} \mathrm{ha}^{-1}\right)$.

A produtividade brasileira média de alho na safra de 2012 foi de $10.601 \mathrm{~kg} \mathrm{ha}^{-1}$ (CONAB, 2013). A produtividade média de bulbos obtida neste estudo foi inferior à produtividade média nacional (Tabela 3). Estes resultados podem ser atribuídos à grande sensibilidade da cultura do alho às condições edafoclimáticas de cultivo, como fotoperíodo, temperatura, altitude. Sedoguchi (2002) constatou que os cultivares Quitéria e Caçador não formaram bulbos devido à temperatura elevada e a baixa altitude da região de Seropédica (RJ). Neste contexto, é de grande importância a realização de estudos com a cultura do alho na região sudeste do Estado de Mato Grosso para que cultivares mais adaptados possam ser posicionadas.

Tabela 3. Produtividade de bulbos das diferentes cultivares de alho cultivados na região sudeste do estado de Mato Grosso. IFMT Campus São Vicente, Santo Antônio de Leverger, 2012

\begin{tabular}{lc}
\hline Cultivares & Produtividade $\left(\mathbf{k g ~ h a} \mathbf{~}^{-1}\right)$ \\
\hline Quitéria L.V (vernalizado) & $4.535, \mathrm{a}$ \\
Quitéria (vernalizado) & $4.107 \mathrm{a}$ \\
Caçador (vernalizado) & $3.882 \mathrm{a}$ \\
Hozan & $3.792 \mathrm{a}$ \\
Jonas (vernalizado) & $3.740 \mathrm{a}$ \\
Curitibanos & $2.912 \mathrm{~b}$ \\
Chinês Real & $2.785 \mathrm{~b}$ \\
Gigante do Núcleo & $2.565 \mathrm{~b}$ \\
Caturra & $2.507 \mathrm{~b}$ \\
Amarante L.V & $2.387 \mathrm{~b}$ \\
Hozan L.V & $2.332 \mathrm{~b}$ \\
Amarante & $2.040 \mathrm{c}$ \\
Gigante Roxo & $1.460 \mathrm{c}$ \\
\hline CV (\%): 14,94 & \\
Média Geral: 2.974 & \\
Médias seguidas pela mesma letra, na coluna, pertencem ao mesmo grupo pelo \\
teste de agrupamento de Scott e Knott (1974), a 5\% de probabilidade.
\end{tabular}




\section{Conclusões}

O cultivar Quitéria L.V (vernalizado) apresentou o maior diâmetro e massa média de bulbos quando comparado aos demais cultivares testados.

As maiores produtividades totais de bulbos foram obtidas para os quatro cultivares submetidos a um período de vernalização pré-plantio (Quitéria L.V, Quitéria, Caçador e Jonas) e para o cultivar Hozan não vernalizado.

O plantio do cultivar Quitéria L.V., utilizando-se a vernalização pré-plantio do bulbilho-semente, é o mais recomendado para a região sudeste de Mato Grosso, devido a sua maior adaptabilidade às condições edafoclimáticas da região.

\section{Referências Bibliográficas}

CONAB - Companhia Nacional do Abastecimento. Acompanhamento da safra 2012: Conjuntura mensal. Brasília-DF: CONAB, 2013. Disponível em: < http://www.conab.com.br >. Acesso em: 16 jan. 2013.

FILGUEIRA, F. A. R. Novo manual de olericultura: agrotecnologia moderna na produção e comercialização de hortaliças. Viçosa-MG: UFV, 2003. 402 p.

IBGE. Instituto Brasileiro de Geografia e Estatística. Levantamento sistemático da produção agrícola. Brasília-DF: IBGE, 2015. Disponível em: < http://www.ibge.gov.br > Acesso em: 05 out. 2015.

JONES, M. A.; MANN, L. K. Onion and their allies. New York-USA: Interscience, 1963. 286 p.

MENEZES, S. J. A.; CHARCHAR, J. M.; ARAGÃO, F. A. S. Caracterização morfológica de germoplasma de alho por análises multivariada, componentes principais e variáveis canônicas. Horticultura Brasileira, Brasília-DF, v. 17, n. 2 , p. 96-101, 1999.
MOTA J. H.; YURI J. E.; RESENDE G. M.; SOUZA R. J. Similaridade genética de cultivares de alho pela comparação de caracteres morfológicos, físico-químicos, produtivos e moleculares. Horticultura Brasileira, Brasília-DF, v. 24, n. 2, p. 156-160. 2006.

OLIVEIRA, C, M.; SOUZA, R. J.; YURI, J. E.; MOTA, J. H.; RESENDE, G. M. Determinação do ponto de colheita na produção de alho. Horticultura Brasileira, Brasília-DF, v.21, n.3, p. 506-509, 2003.

OLIVEIRA, F. L. L.; DORIA, H.; TEODORO, R. B.; RESENDE, F. V. Características agronômicas de cultivares de alho em Diamantina. Horticultura Brasileira, Brasília-DF, v. 28, n. 3. p. 355-359. 2010.

SCOTT, A. J.; KNOTT, M. A. A cluster analysis method for grouping means in the analysis of variance. Biometrics, Raleigh, v. 30, n. 3, p. 507-512. 1974.

SEDOGUCHI, E. T.; CARMO, M. G. F.; PARRAGA, M. S. Características morfológicas, de produção e efeitos da vernalização sobre cultivares de alho em duas épocas de plantio em Seropédica-RJ. Agronomia, Seropédica-RJ, v.36, n. 2, p. 42-47, 2002.

TRANI, P. S.; FOLTRAN, D. E.; CAMARGO, M. S.; TIVELLI, S. W.; PASSOS, F. A. Produtividade de Cultivares de Alho na Região Paulista de Tietê. Bragantia, CampinasSP, v. 67, n.3, p. 713-716, 2008.

VILELA N. J; HENZ G. P. Situação atual da participação das hortaliças no agronegócio brasileiro e perspectivas futuras. Cadernos de Ciência \& Tecnologia, Brasília-DF, v. 17, n. 1, p. 71-89, 2000.

ZINK, F. W. Rate of growth and nutrient absorption of late garlic. In: Proceedings of the American Society for Horticultural Science, Geneva-NY, v. 83, n. 579-584, p. 162, 1963. 